\title{
Laryngitis after inhalation of liposomal amikacin
}

\author{
Atsuho Morita ${ }^{1}$, Ho Namkoong ${ }^{2}$, Makoto Hosoya $^{2}$, and Naoki Hasegawa ${ }^{2}$ \\ ${ }^{1}$ Keio University School of Medicine Department of Internal Medicine \\ ${ }^{2}$ Keio University School of Medicine Graduate School of Medicine
}

November 13, 2021

\begin{abstract}
A 56-year-old female with pulmonary Mycobacterium avium complex disease started to inhale of liposomal amikacin. One month later, she developed hoarseness and was diagnosed with laryngitis. The laryngitis healed immediately after discontinuing the treatment, and no recurrence occurred even after resuming intermittent inhalation.

Laryngitis after inhalation of liposomal amikacin

Atsuho Morita M.D. ${ }^{1}$, Ho Namkoong M.D., Ph.D. MPH $^{2}$, Makoto Hosoya M.D., Ph.D. ${ }^{3}$, and Naoki Hasegawa M.D., Ph.D. ${ }^{2}$

${ }^{1}$ Division of Pulmonary Medicine, Department of Medicine, Keio University School of Medicine, Tokyo, Japan.

${ }^{2}$ Department of Infectious Diseases, Keio University School of Medicine, Tokyo, Japan.

${ }^{3}$ Department of Otorhinolaryngology, Head and Neck Surgery, Keio University School of Medicine, Tokyo, Japan.
\end{abstract}

Corresponding author:

Ho Namkoong, M.D., Ph.D., MPH

Department of Infectious Diseases, Keio University School of Medicine, 35 Shinanomachi, Shinjuku, Tokyo 160-8582, Japan

\section{Keywords}

Laryngitis; hoarseness; amikacin liposomal inhalation suspension (ALIS);Mycobacterium avium complex (MAC);

Written informed consent was obtained from the patient to publish this report in accordance with the journal's patient consent policy.

\section{ABSTRACT}

A 56-year-old female with pulmonary Mycobacterium avium complex disease started to inhale of liposomal amikacin. One month later, she developed hoarseness and was diagnosed with laryngitis. The laryngitis healed immediately after discontinuing the treatment, and no recurrence occurred even after resuming intermittent inhalation.

(43 words)

\section{KEY CLINICAL MESSAGE}


Inhalation of liposomal amikacin may cause laryngitis and associated hoarseness, although rapid improvement can be expected by discontinuing treatment. The intermittent inhalation may be effective in resume without recurrence.

(29 words)

\section{CASE DESCRIPTION}

A 56-year-old female patient had been on medication for macrolide-resistant pulmonary Mycobacterium avium complex disease, and had persistently had positive sputum cultures. Because the minimal inhibitory concentration (MIC) of amikacin was sensitive at $4 \mu \mathrm{g} / \mathrm{ml}$, She started amikacin liposomal inhalation suspension (ALIS) in combination with rifampicin and ethambutol. Thereafter, the amount of bacteria in the sputum smear gradually decreased, the cough and sputum were under control. However, hoarseness appeared one month after treatment initiation. Fiberoptic laryngoscopy showed redness and swelling of both vocal cords, indicating laryngitis (Figure 1, black arrow). ALIS therapy was discontinued, and hoarseness improved within a few days. After two weeks, the treatment was resumed and administered every other day. There was no recurrence of hoarseness until five weeks after the resumption, at which point laryngitis had healed (Figure 2, white arrow). There was no laryngeal edema consistently.

It has been reported that dysphonia occurs in $46.6 \%$ of patients subjected to daily ALIS (1), but can improve with a temporary reduction in ALIS inhalation frequency (2). In the present case, the vocal cord morphologically returned to normal after seven weeks of dose reduction. This report suggests that, in case hoarseness appears, intermittent inhalation could permit healing while continuing treatment.

(199 words)

\section{Acknowledgments}

This study was supported by AMED (21fk0108621h0001) and JSPS Grant-in-Aid for Young Scientists (21K15667).

\section{Ethics statement}

This paper was written after obtaining informed consent, and the procedures followed were in accordance with the Declaration of Helsinki.

\section{Conflict of interests}

The authors state that they have no conflict of interest.

\section{Data availability}

The data will not be shared with patient confidentiality.

\section{REFERENCES}

1 Griffith DE, Eagle G, Thomson R, et al. Amikacin Liposome Inhalation Suspension for TreatmentRefractory Lung Disease Caused by Mycobacterium avium Complex (CONVERT). A Prospective, OpenLabel, Randomized Study. Am J Respir Crit Care Med 198(12): 1559-1569, 2018.

2 Swenson C, Lapinel NC, Ali J. Clinical Management of Respiratory Adverse Events Associated With Amikacin Liposome Inhalation Suspension: Results From a Patient Survey. Open Forum Infect Dis 7(4): ofaa079, 2020.

\section{Hosted file}

Images_CCR_AM.docx available at https://authorea.com/users/445942/articles/545322-laryngitisafter-inhalation-of-liposomal-amikacin 